\title{
Probabilistic Anatomical Connectivity Using Completion Fields
}

\author{
Parya MomayyezSiahkal and Kaleem Siddiqi \\ Centre for Intelligent Machines, School of Computer Science, McGill University \\ \{pmamay, siddiqi\}@cim.mcgill.ca
}

\begin{abstract}
Diffusion magnetic resonance imaging has led to active research in the analysis of anatomical connectivity in the brain. Many approaches have been proposed to model the diffusion signal and to obtain estimates of fibre tracts. Despite these advances, the question of defining probabilistic connectivity indices which utilize the relevant information in the diffusion MRI signal to indicate connectivity strength, remains largely open. To address this problem we introduce a novel numerical implementation of a stochastic completion field algorithm, which models the diffusion of water molecules in a medium while incorporating the local diffusion MRI data. We show that the approach yields a valid probabilistic estimate of connectivity strength between two seed regions, with experimental results on the MICCAI 2009 Fibre Cup phantom[1].
\end{abstract}

Keywords: Diffusion-MRI, Tractography, Connectivity, 3D stochastic completion field.

\section{Introduction}

During the past decade advances in diffusion magnetic resonance imaging have led to the development of a variety of tractography algorithms which reconstruct connectivity patterns between distinct cortical and subcortical areas in the human brain. The early deterministic tractography methods, based on streamline tracking and diffusion tensor imaging [2, verified the inherent potential of diffusion-MRI to capture anatomical connectivity non-invasively. However, these approaches suffered from the limitation that they did not explicitly consider the effects of partial volume averaging, the underlying noise in the data and other imaging artifacts. More recent probabilistic tractography algorithms [345] have attempted to address these concerns by providing an approximation of the underlying noise and incorporating it into the fibre propagation framework. Whereas some of these latter schemes employ Monte-Carlo simulations to perform tractography for many iterations [3], others use front propagation approaches to evolve a surface from a seed region and then find the minimum cost trajectories between each voxel and the seed region by back propagating through a "time of arrival" map [4]. In these approaches a measure of connectivity is either defined based on the number of times a voxel has been passed through [36] or by considering the lowest confidence value of all tract segments along a reconstructed 
streamline 44. In a related but distinct approach, Fletcher et al. propose a front evolution scheme to compute a connectivity measure between two regions of interest without explicitly obtaining fibre tracts first [7].

A different class of algorithms tackles the problem of fibre tractography and white matter connectivity by solving a partial differential equation (PDE) and finding the minimal path given the PDE 89. O'Donnell et al. use diffusion tensor estimation and solve for the steady state concentration of the diffusion equation governed by the Fick's law in an anisotropic medium. A Riemannian metric is then used to compute geodesic distances between two points, which in turn is interpreted as their degree of connectivity. Batchelor et al. improve this framework by adding a convection term which incorporates a measure of the anisotropy in the diffusion MRI data at each voxel. The main advantage of these algorithms is their potential to incorporate all the relevant information contained in orientation distribution functions (ODFs), or any other diffusion model, into the tractography framework.

In work that is somewhat distinct from the above methods Momayyez et al. have modeled the Brownian motion of water molecules by a probabilistic 3D random walk, leading to an application of stochastic completion fields to fibre tractography 10. While this framework is also PDE-based, the given PDE describes the evolution of the probability distribution of particles following the random walk, as indicated by the Fokker-Planck equation. The present article extends their results by developing a new, unconditionally stable numerical implementation, which allows for the local diffusion data to be incorporated directly into the stochastic completion field framework. The extension allows for probabilistic estimates of fibre tracts between two seed regions with the property that connectivity strength emerges as an inherent characteristic. We validate the new approach by comparing it with a state-of-the-art probabilistic tractography algorithm on the MICCAI 2009 Fiber Cup Phantom [1].

\section{3D Stochastic Completion Fields}

Using an extension of a model introduced by Williams and Jacobs [11, a 3D stochastic completion field represents the probability that a particle undergoing Brownian motion passes through a given location $(x, y, z)$ with orientation $(\theta, \phi)$ in $3 \mathrm{D}$, while completing a path between two seed regions. In a 3D random walk, particles tend to travel in straight lines with slight changes in orientation controlled by two successive deviations in the osculating and binormal planes, which are respectively proportional to the diffusion parameters $\sigma_{\phi}^{2}$ and $\sigma_{\theta}^{2}$. Additionally, a fraction of particles $\left(1-e^{-\frac{1}{\zeta}}\right)$ decays per unit time step. While $\sigma_{\phi}$ and $\sigma_{\theta}$ maintain the prior assumption of smoothness, the latter decay rate $\zeta$ gives more support to shorter paths. Furthermore, the inherent Markov assumption of the 3D random walk leads to a notion of a stochastic source field and a stochastic sink field. The former represents the probability of passing through any state $(x, y, z, \theta, \phi)$ for a particle which begins in a source state $\left(x_{p}, y_{p}, z_{p}, \theta_{p}, \phi_{p}\right)$, while the latter represents the probability of reaching a final 
sink state $\left(x_{q}, y_{q}, z_{q}, \theta_{q}, \phi_{q}\right)$ from any initial state. The stochastic completion field is computed by scalar multiplication of the source and sink fields.

While Monte-Carlo simulation provides a direct way to compute the stochastic completion field, a more efficient and powerful alternative is to discretize the Fokker-Planck equation which expresses the evolution in time of the probability density function of the particles analytically [10]:

$$
\frac{\partial P}{\partial t}=-\sin \theta \cos \phi \frac{\partial P}{\partial x}-\sin \theta \sin \phi \frac{\partial P}{\partial y}-\cos \theta \frac{\partial P}{\partial z}+\frac{\sigma_{\phi}^{2}}{2} \frac{\partial^{2} P}{\partial \phi^{2}}+\frac{\sigma_{\theta}^{2}}{2} \frac{\partial^{2} P}{\partial \theta^{2}}-\frac{1}{\zeta} P .
$$

Here $P$ is the probability that a particle passes through state $(x, y, z, \theta, \phi)$ in the course of its random walk from a source to a sink region.

Whereas the results in [10] demonstrate a type of proof of concept, they are based on a simple first-order finite difference scheme to solve for the final steady-state distribution of $P$, and as such are quite limited. In particular, such a numerical scheme suffers from instability for complicated fibre pathways with highly curving or twisting tracts. Moreover, the estimation of the completion field in 10] is performed in a manner that is independent of the local diffusion-MRI data, with the final connectivity pattern being obtained by post-multiplication in a Bayesian framework. The Bayesian approach further requires empirical adjustments of parameters which is a significant challenge without prior knowledge of the fibre pathways being estimated.

\section{An Unconditionally Stable Numerical Scheme}

The Fokker-Planck equation given by Eq. 1 is a multi-dimensional PDE which can be solved by implementing an operator splitting strategy, where each time unit is divided into six subunits, each of which formulates separate numerical scheme for each variable. When performing operator splitting, it is usually sufficient to have stable numerical estimation for each operator in order to make the overall scheme stable. For the $x, y$ and $z$ dimensions which are included in the advection part of Eq. 1, we propose to use the Lax-Wendroff scheme which is second-order accurate in time and space. Since in Eq. 1 all the advection coefficients $(-\sin \theta \cos \phi,-\sin \theta \sin \phi,-\cos \theta)$ are always smaller than or equal to one, instability is not an issue. The main challenge in terms of numerical estimation is for the $\sigma$ (diffusion) terms. These terms can get large for fibre tracts with highly curved or twisted segments. To handle this we propose the use of the more complex implicit and unconditionally stable Crank-Nicholson numerical scheme. The numerical estimation is again second order in time and space but because of its implicit character, it involves matrix computation. However the method eliminates the need for thresholds on maximum curvature or torsion values and makes the local incorporation of the diffusion MRI data into the framework possible. The overall numerical scheme is summarized by the following set of equations, where $t$ denotes the time parameter:

$$
\begin{aligned}
P_{x, y, z, \theta, \phi}^{t+\frac{1}{6}} & =P_{x, y, z, \theta, \phi}^{t}-\sin \theta \cos \phi \frac{\Delta t}{2 \Delta x}\left(P_{x+\Delta x, y, z, \theta, \phi}^{t}-P_{x-\Delta x, y, z, \theta, \phi}^{t}\right) \\
& +\frac{(\sin \theta \cos \phi)^{2} \Delta t^{2}}{2 \Delta x^{2}}\left(P_{x+\Delta x, y, z, \theta, \phi}^{t}-2 P_{x, y, z, \theta, \phi}^{t}+P_{x-\Delta x, y, z, \theta, \phi}^{t}\right)
\end{aligned}
$$




$$
\begin{aligned}
P_{x, y, z, \theta, \phi}^{t+\frac{1}{3}}= & P_{x, y, z, \theta, \phi}^{t+\frac{1}{6}}-\sin \theta \sin \phi \frac{\Delta t}{2 \Delta y}\left(P_{x, y+\Delta y, z, \theta, \phi}^{t+\frac{1}{6}}-P_{x, y-\Delta y, z, \theta, \phi}^{t+\frac{1}{6}}\right) \\
+ & \frac{(\sin \theta \sin \phi)^{2} \Delta t^{2}}{2 \Delta y^{2}}\left(P_{x, y+\Delta y, z, \theta, \phi}^{t+\frac{1}{6}}-2 P_{x, y, z, \theta, \phi}^{t+\frac{1}{6}}+P_{x, y-\Delta y, z, \theta, \phi}^{t+\frac{1}{6}}\right) \\
P_{x, y, z, \theta, \phi}^{t+\frac{1}{2}}= & P_{x, y, z, \theta, \phi}^{t+\frac{1}{3}}-\cos \theta \frac{\Delta t}{2 \Delta z}\left(P_{x, y, z+\Delta z, \theta, \phi}^{t+\frac{1}{3}}-P_{x, y, z-\Delta z, \theta, \phi}^{t+\frac{1}{3}}\right) \\
+ & \frac{(\cos \theta)^{2} \Delta t^{2}}{2 \Delta z^{2}}\left(P_{x, y, z+\Delta z, \theta, \phi}^{t+\frac{1}{3}}-2 P_{x, y, z, \theta, \phi}^{t+\frac{1}{3}}+P_{x, y, z-\Delta z, \theta, \phi}^{t+\frac{1}{3}}\right) \\
P_{x, y, z, \theta, \phi}^{t+\frac{2}{3}}- & \frac{\sigma_{\phi}^{2} \Delta t}{4(\Delta \phi)^{2}}\left(P_{x, y, z, \theta, \phi+\Delta \phi}^{t+\frac{2}{3}}-2 P_{x, y, z, \theta, \phi}^{t+\frac{2}{3}}+P_{x, y, z, \theta, \phi-\Delta \phi}^{t+\frac{2}{3}}\right)= \\
& P_{x, y, z, \theta, \phi}^{t+\frac{1}{2}}+\frac{\sigma_{\phi}^{2} \Delta t}{4(\Delta \phi)^{2}}\left(P_{x, y, z, \theta, \phi+\Delta \phi}^{t+\frac{1}{2}}-2 P_{x, y, z, \theta, \phi}^{t+\frac{1}{2}}+P_{x, y, z, \theta, \phi-\Delta \phi}^{t+\frac{1}{2}}\right) \\
P_{x, y, z, \theta, \phi}^{t+\frac{5}{6}}- & \frac{\sigma_{\theta}^{2} \Delta t}{4(\Delta \theta)^{2}}\left(P_{x, y, z, \theta+\Delta \theta, \phi}^{t+\frac{5}{6}}-2 P_{x, y, z, \theta, \phi}^{t+\frac{5}{6}}+P_{x, y, z, \theta-\Delta \theta, \phi}^{t+\frac{5}{6}}\right)= \\
& P_{x, y, z, \theta, \phi}^{t+\frac{2}{3}}+\frac{\sigma_{\theta}^{2} \Delta t}{4(\Delta \theta)^{2}}\left(P_{x, y, z, \theta+\Delta \theta, \phi}^{t+\frac{2}{3}}-2 P_{x, y, z, \theta, \phi}^{t+\frac{2}{3}}+P_{x, y, z, \theta-\Delta \theta, \phi}^{t+\frac{2}{3}}\right) \\
P_{x, y, z, \theta, \phi}^{t+1}= & e^{-\frac{1}{\zeta} P_{x, y, z, \theta, \phi}^{t+\frac{5}{6}} \cdot}
\end{aligned}
$$

It is clear from Eq.2 that the computation at each voxel is local and hence allows for parallelization and reduction in time complexity of the algorithm. In addition, for every voxel distinct diffusion $\sigma_{\theta}, \sigma_{\phi}$ and decay $\zeta$ coefficients can be specified using properties of the local diffusion-MRI data. Thus, the applicability of the stochastic completion field model to fibre tractography is significantly extended. We re-emphasize that although the new numerical scheme is computationally more complex than that of 10] (the run time increases by approximately 40\%), the method in [10] has limited practical utility for curved fibre pathways.

\section{Local Incorporation of Diffusion-MRI Data}

To tune the diffusion parameters $\sigma_{\phi}, \sigma_{\theta}$ and the decay parameter $\zeta$ locally, we use information from the fibre orientation distribution (FOD) function at each voxel, calculated using the approach of Anderson [12. The basic idea is to decrease diffusion (and correspondingly increase the likelihood of trajectories) in directions that are aligned with fiber orientations supported by the data. Specifically, $\sigma_{\phi}$ and $\sigma_{\theta}$ are determined by finding the closest FOD maximum to each $(\phi, \theta)$ direction and computing the two angular differences between a given direction and the chosen maximum. The local corresponding $\sigma_{\phi}$ and $\sigma_{\theta}$ values are then set equal to these angular differences. The decay rate $\zeta$ is also chosen based on the angular difference between a given direction $(\phi, \theta)$ and the chosen FOD maximum. When the angular difference is greater than $\pi / 4$, the decay coefficient is made very small to suppress trajectories in directions that are far from the FOD maxima. When the $(\phi, \theta)$ direction closely matches the FOD maximum, a fixed decay coefficient is assigned to it. Overall this strategy allows parameters 
to be set automatically from the FODs and it supports smoothly varying fibre tracts while avoiding an excessive penalty for curved fibre pathways 1

To reconstruct the connectivity pattern, two independent probability distributions are evolved based on Eq. 1. In the first, the particles are initially placed in one seed region to generate the source field. In the second the particles are constrained to end in the other seed region to generate the sink field. The final stochastic completion field, which is a probabilistic representation of the connectivity pattern, is given by the multiplication of these two fields.

\section{Connectivity Strength}

Defining an index of anatomical connectivity is itself a topic of great interest in the neuroscience community. Most of the connectivity measures proposed thus far in the literature are based on tractography algorithms. Two of the most commonly used connectivity measures reported in the literature consists of: (1) voxel counting: counting the number of times an ROI has been reached from a seed region when employing a Monte-Carlo simulation 35,6] or, (2) finding the weakest link: assigning a weight to each tract segment along a reconstructed fibre pathway based on some weight function or on the previous Monte-Carlo approach and considering the lowest confidence value of all tract segments along the streamline [4. A limitation of these methods is that they are heuristic in nature. They do not incorporate relevant information along the reconstructed fibre tract into the connectivity measure in a principled fashion. They also suffer from reduced connectivity values with distance from the seed region.

The stochastic completion field model offers a potential solution. The algorithm's output is the steady-state probability distribution of all the states when joining the source and sink regions. In other words, the probability of reaching each state has been calculated during the evolution of the probability distributions from the source and the sink regions while integrating all the computed probabilities along the connectivity pattern. Consequently, the probability values computed for the source and sink ROIs during the computation actually represent the measure of connectivity between them. Moreover, choosing the decay rate based on the approximate length of the fibre tract eliminates the length dependency issue. To obtain the connectivity strength between the source and sink ROIs, it is therefore sufficient to compute the average probability values of all the voxels included in these regions.

\footnotetext{
${ }^{1}$ Ultimately, in order to make the results obtained for different fibre tracts comparable, the decay rate has to be chosen relative to the length of each fibre. To accomplish this the algorithm is run once using a decay rate proportional to the Euclidean distance between the source and sink regions. Following this run, the obtained connectivity pattern is used to approximate the length of the underlying fibre tracts. This length is then used to set the fixed decay rate for a second run of the algorithm.
} 


\section{Experiments}

\subsection{Diffusion-MRI Phantom Data}

The proposed algorithm was tested on the MICCAI 2009 Fibre cup phantom 1, for which ground truth fibre tracts are available. The phantom is made of hydrophobic acrylic fibres whose diameter is of the same scale as that of myelinated axon:2. Two diffusion-MRI datasets have been provided with different spatial resolutions of $3 \times 3 \times 3 \mathrm{~mm}^{3}$ and $6 \times 6 \times 6 \mathrm{~mm}^{3}$ of which we have selected the higher resolution images with an associated $b$-value of $1500 \mathrm{~s} / \mathrm{mm}^{2}$.

\subsection{Results}

The results of the algorithm for six different source and sink region pairs are shown in Fig. 1. which depicts the steady-state probability distribution for each pair. Based on the ground truth, it is known that the first four pairs are associated with a true fibre tract while the latter two are not. For the purpose of comparison, we have implemented a probabilistic fiber tractography algorithm similar to that of [5], where a residual bootstrapping scheme is first used to estimate the uncertainty of a voxels FOD using a single HARDI acquisition. The estimated uncertainties are then used in a Monte-Carlo simulation of a probabilistic streamline tractography algorithm. Confidence values are also assigned to the reconstructed streamlines using a weakest link approach. The probabilistic streamline tractography was run for 500 iterations, with a seed frequency of 10 per voxel for all ROIs and a threshold of $75^{\circ}$ for the maximum turning angle from one voxel to the next. The latter threshold was optimized empirically.

Table 1 summarizes the connectivity indices obtained from our algorithm and from probabilistic tractography. The connectivity index for each scenario is computed by taking the average of the corresponding values for all the voxels included in the sink and source ROIs.

Table 1. Connectivity indices obtained for six different seed region pairs

\begin{tabular}{c||cccccc} 
Algorithm & $(\mathrm{a})$ & $(\mathrm{b})$ & (c) & (d) & (e) & (f) \\
\hline \hline Stochastic Completion Field & 0.17152 & 0.22445 & 0.04716 & 0.24342 & 0.00551 & 0.07559 \\
Probabilistic Tractography & 0.00008 & 0.24051 & 0.00819 & 0 & 0 & 0
\end{tabular}

There are several observations that can be made from Table 1, First, the connectivity indices obtained from probabilistic tractography span a wide range, which can be due to the heuristic nature of the algorithm and its dependency on length. Moreover, while the algorithm assigns a connectivity index of zero to cases (e) and (f), which are not associated with a true fibre pathway, it is also not capable of finding the true connectivity for case (d), which is a straight fibre tract and should be an easy pathway to reconstruct. The connectivity

${ }^{2}$ For details, visit http://www.Inao.fr/spip.php?article107 


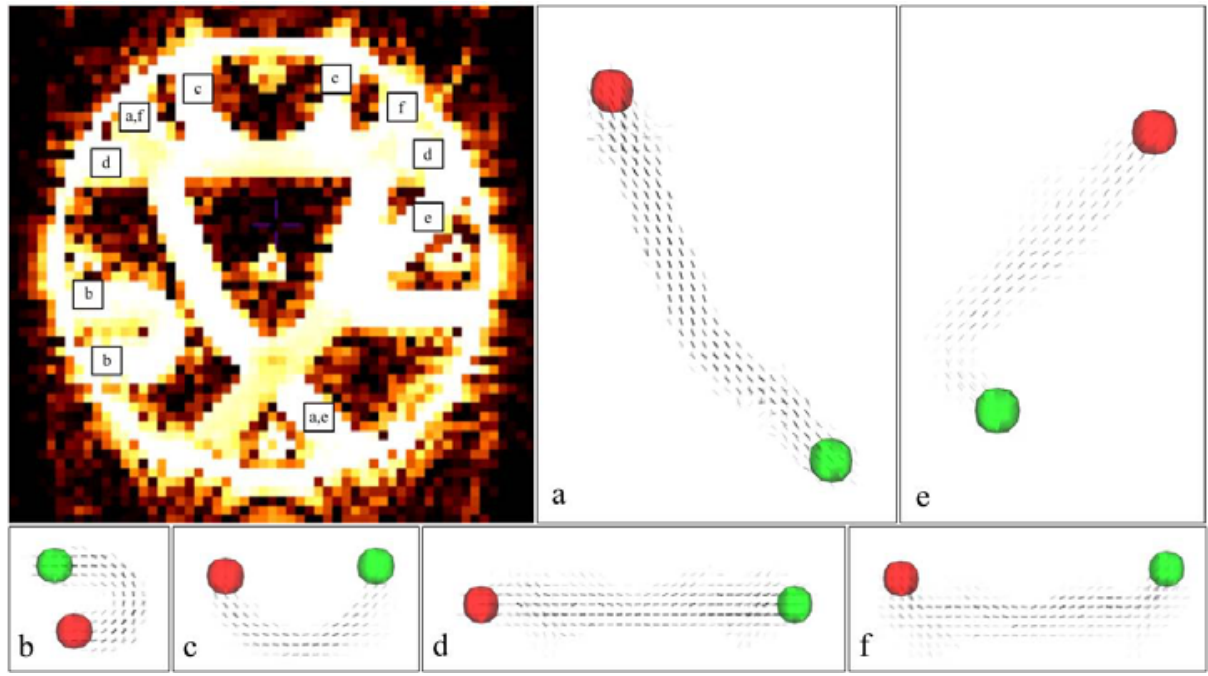

Fig. 1. Results of the local completion field algorithm. The probability of a state is inversely proportion to the transparency of the associated orientation (The reader is encouraged to zoom-in on the electronic version to get a better sense of the 3D characteristics). Top-Left: Mean diffusivity image and the seed regions used.

measures obtained by the completion field algorithm, on the other hand, are comparatively in the same range for cases (a), (b) and (d), which represent true connections. The value obtained for case (e) is very small, showing the low confidence of the algorithm in finding a connection. For cases (c) and (f), however, the algorithm fails to assign a higher connectivity to tract (c). This is due to the fact that the true fibre tracts form a bottle neck with another pathway along the streamline. As a result, at a local scale the algorithm favors the smoother connections leading to a lower connectivity value for case (d) and a higher value for case (f). This failure can be essentially solved by acquiring higher resolution data. In the meantime, one of our future goals is to modify the algorithm to incorporate constraints such as curvature consistency to be able to handle such ambiguous situations.

\section{Conclusion}

We have addressed the problem of anatomical connectivity by extending the stochastic completion field algorithm introduced in [10. A new, unconditionally stable numerical implementation has been developed which allows for the local incorporation of the diffusion data into the framework. The extension provides a basis for theoretically derived measures of connectivity based on probabilistic estimates of fibre tracts. The results obtained on the phantom demonstrate the inherent ability of the algorithm to compute consistent connectivity measures and to reconstruct the most probable fibre pathways between two seed regions. 
This approach can find highly curving fibre tracts without the need to impose thresholds on maximum curvature or torsion. The algorithm has low computational complexity, which is independent of the size of the seed regions, and the automatic tuning of its parameters is a further advantage.

\section{References}

1. Poupon, C., Rieul, B., Kezele, I., Perrin, M., Poupon, F., Mangin, J.: New diffusion phantoms dedicated to the study and validation of high-angular-resolution diffusion imaging (HARDI) models. J. Magnetic Resonance in Medicine 60, 1276-1283 (2008)

2. Mori, S., Crain, B.J., Chacko, V.P., Zijl, P.C.M.: Three dimensional tracking of axonal projections in the brain by magnetic resonance imaging. Annals of Neurology 45, 265-269 (1999)

3. Behrens, T.E., Berg, H.J., Jbabdi, S., Rushworth, M.F., Woolrich, M.W.: Probabilistic diffusion tractography with multiple fibre orientations: what can we gain? Neuroimage 34, 144-155 (2007)

4. Parker, G.J.M., Wheeler-Kingshott, C.A.M., Barker, G.J.: Estimating distributed anatomical connectivity using fast marching methods and diffuson tensor imaging. IEEE Transaction on Medical Imaging 21, 505-512 (2002)

5. Berman, J.I., Chung, S., Mukherjee, P., Hess, C.P., Han, E.T., Henry, R.G.: Probabilistic streamline q-ball tractography using the residual bootstrap. NeuroImage 39, 215-222 (2008)

6. Yo, T.S., Anwander, A., Descoteaux, M., Fillard, P., Poupon, C., Knösche, T.R.: Quantifying brain connectivity: A comparative tractography study. In: Yang, G.Z., Hawkes, D., Rueckert, D., Noble, A., Taylor, C. (eds.) MICCAI 2009. LNCS, vol. 5761, pp. 886-893. Springer, Heidelberg (2009)

7. Fletcher, P.T., Tao, R., Jeong, W.K., Whitaker, R.T.: A volumetric approach to quantifying region-to-region white matter connectivity in diffusion tensor MRI. In: Karssemeijer, N., Lelieveldt, B. (eds.) IPMI 2007. LNCS, vol. 4584, pp. 346-358. Springer, Heidelberg (2007)

8. O'Donnell, L., Haker, S., Westin, C.F.: New approaches to estimation of white matter connectivity in diffusion tensor MRI: Elliptic PDEs and geodesics in a tensorwarped space. In: Dohi, T., Kikinis, R. (eds.) MICCAI 2002. LNCS, vol. 2488, pp. 459-466. Springer, Heidelberg (2002)

9. Batchelor, P.G., Hill, D.L.G., Atkinson, D., Calamante, F., Connelly, A.: Fibretracking by solving the diffusion-convection equation. In: Proceedings of Int. Soc. Mag. Reson. Med., Honolulu, vol. 10 (2002)

10. Momayyez, P., Siddiqi, K.: 3D stochastic completion fields for fiber tractography. In: IEEE Workshop on Mathematical Methods in Biomedical Image Analysis (MMBIA 2009), Miami Beach, pp. 178-185 (2009)

11. Williams, L., Jacobs., D.: Stochastic completion fields: A neural model of illusory contour shape and salience. Neural Computation 9, 837-858 (1997)

12. Anderson, A.W .: Measurement of fiber orientation distributions using high angular resolution diffusion imaging. J. Magnetic Resonance in Medicine 54, 1194-1206 (2005) 